\title{
Study of horn flies as vectors of bovine leukemia virus
}

\author{
Carlos Javier Panei ${ }^{1,2, *}$, Alejandra Edith Larsen ${ }^{1}$, Nadia Analía Fuentealba $^{1,2}$, German Ernesto Metz ${ }^{1,2}$, María Gabriela \\ Echeverría $^{1,2}$, Cecilia Mónica Galosi ${ }^{1,3}$ and Alejandro Rafael Valera ${ }^{1}$ \\ ${ }^{1}$ Laboratorio de Virología, Facultad de Ciencias Veterinarias, Universidad Nacional de La Plata, \\ Buenos Aires, Argentina \\ ${ }^{2}$ Comisión Nacional de Investigaciones Científicas y Técnicas (CONICET), Buenos Aires, Argentina \\ ${ }^{3}$ Comisión de Investigaciones Cientificas (CIC), Buenos Aires, Argentina
}

\begin{abstract}
Bovine leukemia virus (BLV) is the agent responsible for enzootic bovine leukosis, the most common neoplastic disease in cattle. The horn fly, a major hematophagous pest of cattle, is able to transmit different diseases in cattle. However, its implication in BLV transmission under a natural environment is still discussed. The objectives of this work were to determine the presence of BLV in horn flies (by sequencing) and to evaluate the ability of horn flies to transmit BLV to cattle (through an experimental assay under a natural environment). To demonstrate the presence of BLV in the flies, 40 horn flies were collected from a BLV-positive cow with a sweep net and 10 pools with four hornfly mouthparts each were prepared. The presence of BLV was determined by nested polymerase chain reaction and sequencing. To demonstrate BLV transmission, other 40 flies were collected from the same BLV-positive cow with a sweep net. Eight homogenates containing five horn-fly mouthparts each were prepared and injected to eight cows of different breeds, and blood samples were collected every 21 days. Then, to evaluate the ability of horn flies to transmit BLV to grazing cattle under natural conditions, both infected and uninfected cattle from the experimental transmission assay were kept together in the same paddock with more than 200 horn flies per animal for 120 days. Blood samples were collected every 20 days and the number of flies was determined. The sequencing results confirmed the presence of the provirus in horn flies. The results also confirmed that BLV transmission is a possible event, at least experimentally. However, the role of horn flies as vectors of BLV under a natural grazing system is still discussed.
\end{abstract}

Keywords: Blood-sucking insect, BLV transmission, Enzootic bovine leukosis, Haematobia irritans, Horn fly.

\section{Introduction}

Bovine leukemia virus (BLV) is the etiologic agent responsible for enzootic bovine leukosis, which is the most common neoplastic disease in cattle. After the infection, the BLV genome integrates as a provirus into the different populations of white blood cells (Panei et al., 2013a). Approximately, 30\% of infected cattle develop persistent lymphocytosis (PL), 0.1\%-5\% may develop tumors that invariably lead to death, and about $65 \%-70 \%$ remain as asymptomatic seropositive carriers in an aleukemic state (AL) (Lagarias and Radke, 1989). BLV is transmitted predominantly by horizontal routes, mostly by transfer of infected cells via bloodcontaminated devices and physical contact (Lassauzet et al., 1991; Kohara et al., 2006). Several researchers have established the role of tabanids, stable flies, and horn flies in BLV transmission under experimental conditions (Buxton et al., 1985; Foil et al., 1988; Ooshiro et al., 2013). However, since in these studies the horn flies were fed "in vitro" on the blood collected from a BLV-infected cow, the role of horn flies under natural conditions of feeding is still uncertain.

The horn fly, Haematobia irritans (Linnaeus 1758) (Diptera: Muscidae), is a major hematophagous pest of cattle in Europe and North and South America (Kuramochi, 2000). These flies are small diptera of dark gray color, and the smallest flies in cattle. Both males and females usually spend their life on the back of the same host, but sometimes also migrate to a new host (Oyarzún et al., 2008). The backs of cattle provide an ideal location for horn flies to feed because this allows them to avoid the head and tail of the animal. Each of these flies can consume between 11 and $21 \mathrm{mg}$ blood/d, feeding 24-38 times/d using their piercing sucking proboscis (Foil and Hogsette, 1994).

The objectives of this work were to determine the presence of BLV in horn flies (by sequencing) and to evaluate the ability of horn flies to transmit BLV to cattle (through an experimental assay and under a natural environment).

\section{Materials and Methods}

Forty horn flies were collected with a sweep net from a donor cow (Holstein: steer \#437), previously detected as BLV-positive by Agar Gel Immunodiffusion (AGID), and nested polymerase chain reaction (PCR) and with PL, in January 2017, a summer with high temperature and humidity. The cutoff value used for

\footnotetext{
*Corresponding Author: Carlos Javier Panei. Laboratorio de Virología, Facultad de Ciencias Veterinarias, Universidad
} 
the classification between PL and AL positive to BLV was 10,000 cells/ $\mu 1$ (Panei et al., 2013b). The flies were immobilized and their mouthparts separated according to that described by Elbers et al. (2013). The mouthparts were divided into 10 pools of 4 mouthparts each. Using a sterile mortar, each pool was macerated and suspended in a $15-\mathrm{ml}$ tube with proteinase K solution (Qiagen, Germany) and then incubated overnight at $37^{\circ} \mathrm{C}$. Genomic DNA was extracted according to the protocols using a commercial kit (DNA Purification Kit, Promega, WI). DNA concentrations were determined using Qubit 2.0 Fluorometric Quantitation (Invitrogen). The primers used and the nested PCR conditions for BLV detection were the same as those described in Licursi et al. (2003).

The amplified products were visualized on $1.5 \%$ agarose gels in tris-borate-EDTA buffer stained with ethidium bromide. The bands were identified according to their size using a 100-bp DNA ladder as a marker (Promega, WI). Each amplified product was purified and sent to the Instituto de Biotecnología of the Instituto Nacional de Tecnología Agropecuaria, Buenos Aires, Argentina for sequencing. The nucleotide sequences obtained were assembled and analyzed by the Bioedit software and the nucleotide compositions were compared with the BLV strains reported in GenBank.

For the experimental infection assay, $12 \mathrm{BLV}$-negative animals (four black Aberdeen Angus animals: heifers $\# 042$, \#242 and \#312 and steer \#383; four red Aberdeen Angus animals: heifers \#246 and \#401 and steers \#313 and \#350; and four Holstein animals: steer \#080 and heifers \#151, \#268 and \#359) and one BLV-positive donor cow (animal \#437), all from a farm called "Don Joaquin" from the Faculty of Veterinary Sciences of the National University of La Plata, Argentina, and aged 24-36 mo old at the time of the experiment, were used. To determine the status of BLV before the assay, the cattle were checked once a month for 6 mo by AGID and nested PCR.

To prepare the inoculum, other 40 horn flies were collected with a sweep net from the BLV-positive donor cow (animal \#437). The flies were immobilized and the mouthparts separated according to that previously described (Elbers et al., 2013). Each inoculum was prepared with five mouthparts each. The mouthparts were placed into $15-\mathrm{ml}$ tubes with $2 \mathrm{ml}$ of Minimum Essential Medium (Gibco ${ }^{\circledR}$ ) solution supplemented with penicillin $(100 \mathrm{U} / \mathrm{ml})$, streptomycin $(100 \mathrm{U} / \mathrm{ml})$, and nystatin $(100 \mathrm{U} / \mathrm{ml})$. Finally, each inoculum was homogenized with an Ultra Turrax T25 Ika homogenizer (Labortechnik, Germany).

Eight of the 12 BLV-negative animals (animals \#042, $\# 080, \# 151, \# 242$, \#246, \#268, \#312, and \#313) were injected in the left axilla with $2 \mathrm{ml}$ of each inoculum, whereas three were kept without inoculation as negative controls (animals \#350, \#359, and \#383) and one (animal \#401) was injected with $5 \mu 1$ of whole blood from the BLV-positive donor cow (animal \#437) as a control of the susceptibility to BLV infection. The animals were marked individually with numbered ear tags. Then, whole blood was collected with and without heparin from all animals at 21-day intervals $(0,21,42,63,84,105$, and $126 \mathrm{~d})$ for antibody and provirus detection. All assays were performed with sterile material.

To evaluate the ability of horn flies to transmit BLV to grazing cattle under natural feeding behavior conditions, infected and uninfected cattle from the experimental transmission assay were kept together with the BLV-positive donor cow in the same paddock for 120 d (from December 2017 to March 2018). Blood samples were collected every $20 \mathrm{~d}$ and the number of horn flies on each animal counted. Serological and molecular detection was carried out as previously described.

\section{Results and Discussion}

Three of the 10 pools (pools 2, 4, and 9) with four horn-fly mouthparts each were positive to BLV, corresponding to a 444-bp fragment by nested PCR (Fig. 1). To corroborate that these PCR products had not been produced due to nonspecific amplification, the three PCR products were sequenced, aligned, and compared with those available at GenBank. The percentage of homology found in the three pools was $100 \%$, while the percentage of identity when compared with sequences reported in South America (Polat et al., 2016) was $99 \%$, confirming the presence of BLV in the flies (Fig. 2).

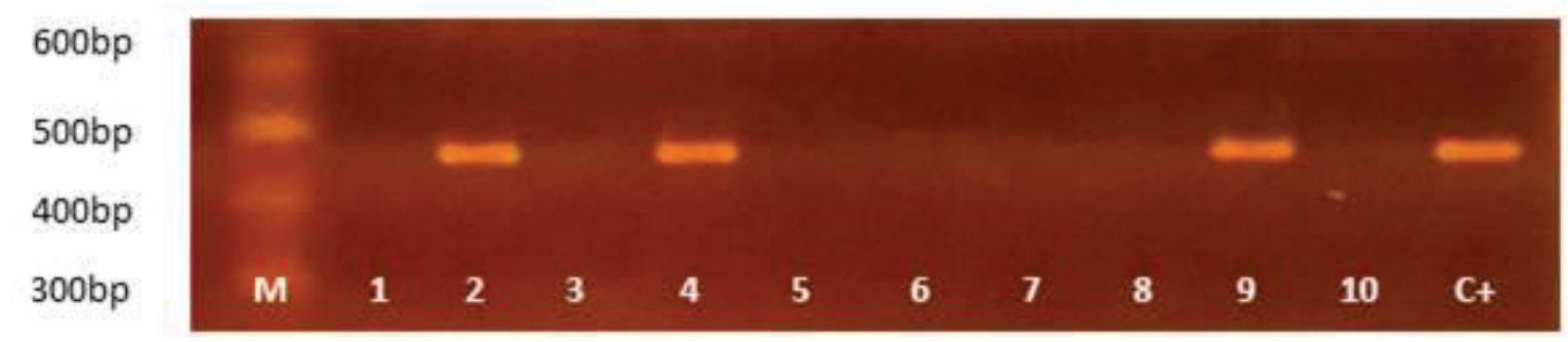

Fig. 1. Agarose gel electrophoresis. M: 100-bp marker, lanes 2, 4, and 9: amplification of a 444-bp fragment corresponding to BLV. C+: positive control. 


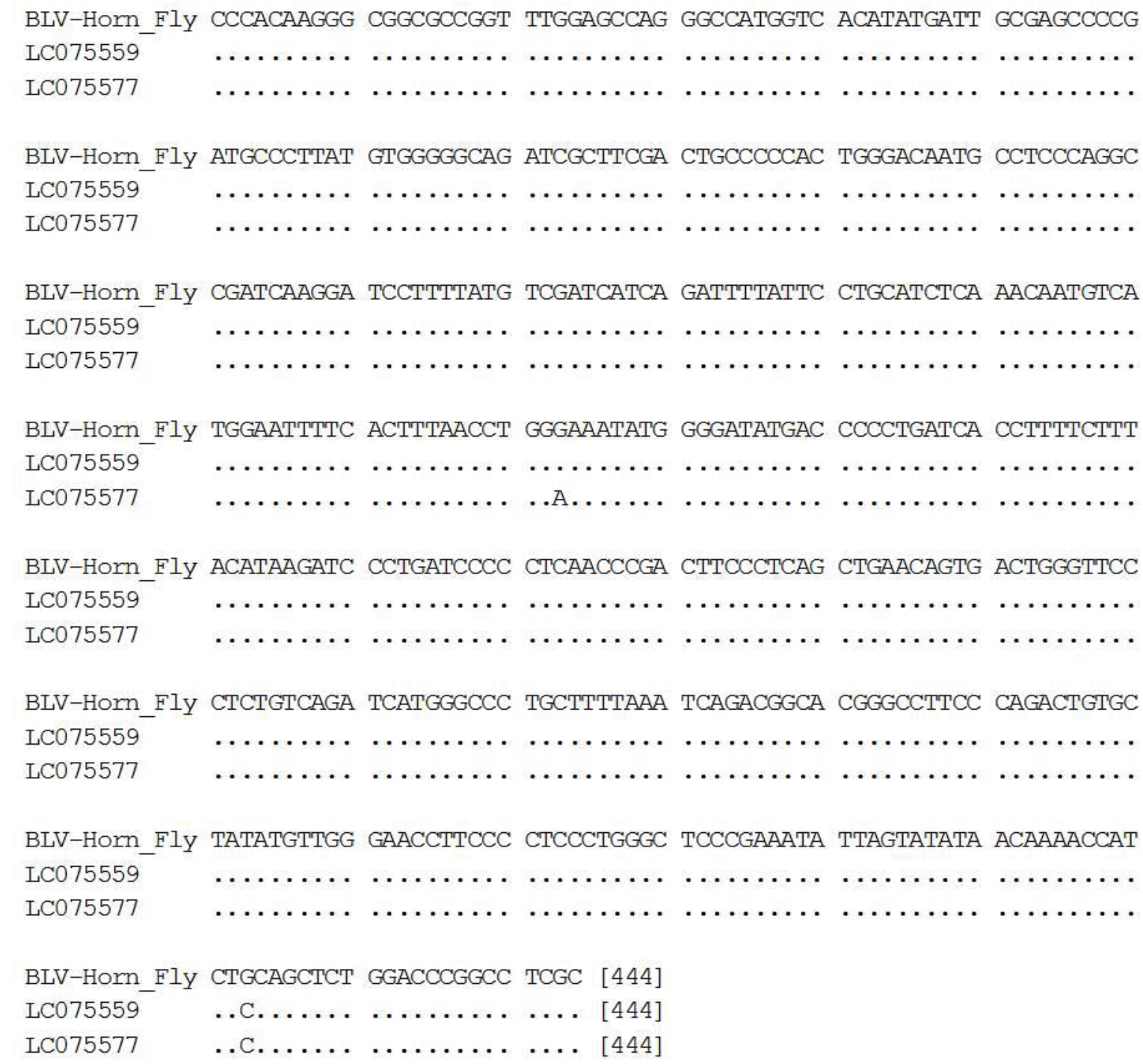

Fig. 2. Nucleotide sequences of 444 bp obtained by PCR from horn flies (BLV-horn-fly) and compared with BLV strains reported in GenBank (LC075559-Paraguay, L075577-Peru, Polat et al., 2016).

After $126 \mathrm{~d}$ of experimental infection assay, in two out of the eight experimentally infected animals (animals \#080 and \#242), the results showed the presence of the virus by AGID detection $63 \mathrm{~d}$ after inoculation. In contrast, in the animal used as a control of the susceptibility to BLV infection (animal \#401), the presence of the virus was detected $42 \mathrm{~d}$ after inoculation.

BLV was also molecularly detected in the same two experimentally infected animals $42 \mathrm{~d}$ after inoculation and in the animal used as a control of the susceptibility to BLV infection $21 \mathrm{~d}$ after inoculation (Fig. 3).

In the experiment carried out by Buxton et al. (1985), infection of calves occurred after inoculation of 100 mouthparts from horn flies removed immediately after feeding them "in vitro" with blood from a BLV-positive cow. In our study, two out of eight cattle were infected with only five mouthparts per inoculum of horn flies captured in their environment under natural feeding behavior and probably having fed with blood from the BLV-positive donor cow (animal \#437) where they were captured. This result seems to represent what happens under a natural grazing system. The results of this study show not only that two animals were BLV-infected, but also that a smaller number of flies captured from a natural environment were enough to induce the experimental infection. Despite this, six of the animals remained BLV-negative. A possible explanation for this is that the inocula used to infect these animals did not possess flies carrying the virus in their mouthparts or that the amount of blood was not enough to produce an infection. 

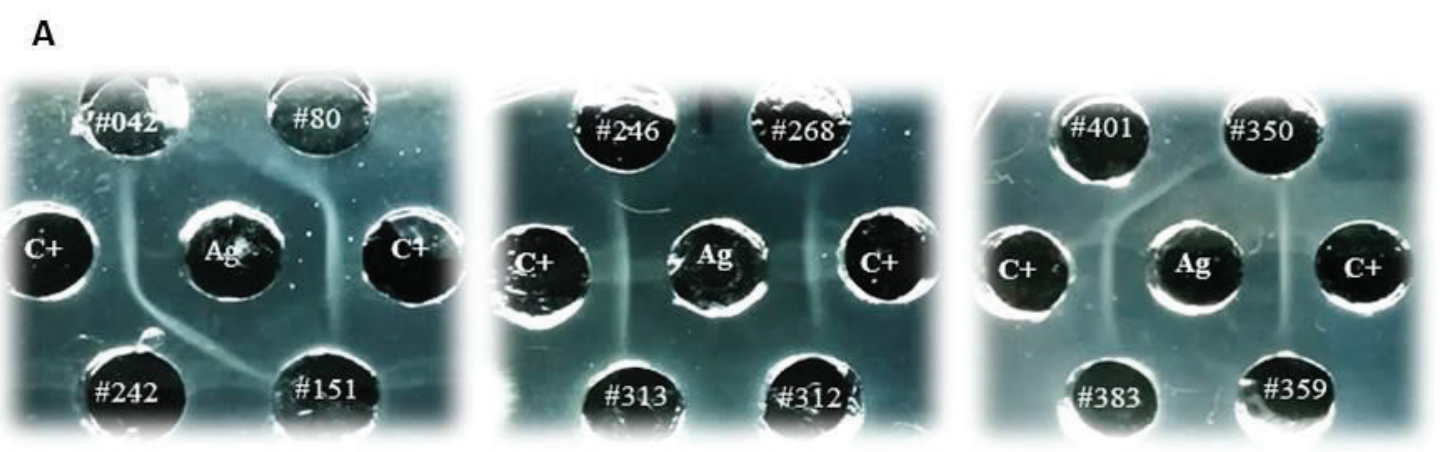

B

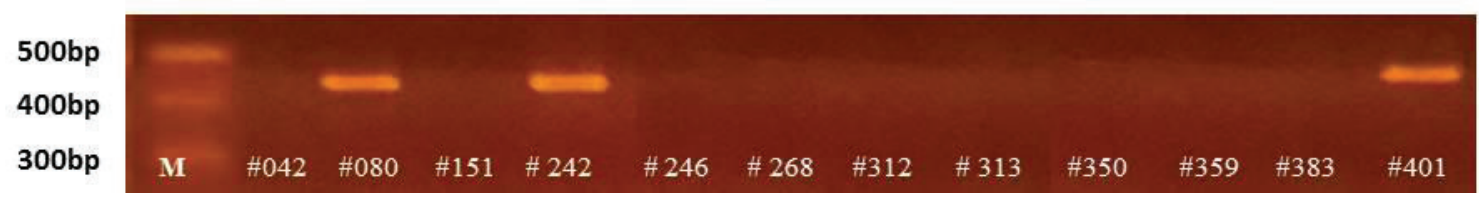

Fig. 3. (A) AGID: Ag: antigen, C+: positive control. Holstein \#80, \#242 and \#401 BLV-positive (precipitation line). (B) Agarose gel electrophoresis. Lane 1: 100-bp marker, lanes 3 (\#80), 5 (\#242) and 13 (\#401): amplification of a 444-bp fragment corresponding to BLV. Lanes 10, 11, and 12: negative controls.

Studies conducted in tabanids have shown that interruption of the feeding is important for efficient mechanical transmission of BLV. It has been estimated that around $10 \%$ of tabanids successfully feed to repletion during their initial attempt of feeding on cattle. If feeding is interrupted, tabanids return to the same or a nearby host within seconds (Mullens, 2002). Thus, another possible explanation of the negative result in six animals could be the fact that the flies present in these inocula been feeding for more than $1 \mathrm{~h}$ before the horn flies were captured. Studies in sheep have shown that when the stable fly mouthparts were removed $1 \mathrm{~h}$ or more after feeding from blood from a BLV-positive cow, the mouthparts inoculated were not infected (Buxton et al., 1985). Regrettably, we were not able to verify by PCR all the inocula to determine the presence of the provirus in the genome of white cells because these inocula should be prepared and inoculated as soon as possible to avoid white cell death.

The prevalence of blood-sucking insects during the summer and the beginning of autumn, when high temperatures and humidity still remain, indicates that these insects spend more time in the environment. Once horn flies locate a herd, they remain on them and rarely leave it. However, the ability of flies to migrate between animals when animals are grouped is a real sanitation problem in BLV infection by blood-sucking insects (Kobayashi et al., 2014). To evaluate the BLV transmission by horn flies under naturally infected (three animals with AL) and uninfected cattle from the experimental infection assay together with the BLVpositive donor cow were kept in the same paddock. More than 200 flies were counted in each animal every $20 \mathrm{~d}$, which implies an important number of insects as potential BLV vectors. However, the result obtained after $120 \mathrm{~d}$ did not evidence the presence of provirus or BLV antibodies by nested PCR and AGID, respectively, in the breeds used in this assay. Probably, the differences in fly blood intake may be partially explained by the variations in skin blood flow and/or epidermal thickness (Breijo et al., 2014), as well as by the hair density and sebaceous secretions (Pruett et al., 2003) in each breed.

Some previous studies have considered that bloodsucking insects are a low risk factor to BLV transmission (Sprecher et al., 1991; Hopkins and DiGiacomo, 1997). However, the factors mentioned above also seem to play a role in the transmission of BLV among cattle. Unlike the BLV-positive donor cow, which presented PL, the three infected animals presented AL. As established by Baldachino et al. (2013) in stable flies, the number of flies, the proportion of infected animals, the PL condition, and the level of proviral load could also be important factors to consider.

The results of the present study confirmed the presence of the provirus in horn flies by sequencing, and the BLV transmission, at least experimentally, with pools of only five mouthparts flies that had been fed with blood from a BLV-positive cow in natural behavior conditions. However, the role of horn flies as vectors of BLV under a natural grazing system remains to be confirmed.

\section{Acknowledgments}

We thank Mr. Claudio Leguizamón, Mr. Eduardo Bereta and Mr. Fabian Mosquera for the technical assistance. 


\section{Conflict of interest}

The authors declare that there is no conflict of interest.

\section{References}

Baldachino, F., Muenworn, V., Desquesnes, M., Desoli, F., Charoenviriyaphap, T. and Duvallet, G. 2013. Transmission of pathogens by Stomoxys flies (Diptera, Muscidae): a review. Parasite 20, 26; doi:10.1051/parasite/2013026

Breijo, M., Rocha, S., Ures, X., Pedrana, G., Alonzo, P. and Meikle, A. 2014. Accessibility of blood affects the attractiveness of cattle to horn flies. Med. Vet. Entomol. 28, 116-118.

Buxton, B.A., Hinkle, N.C. and Schultz, R.D. 1985. Role of insects in the transmission of bovine leukosis virus: potential for transmission by stable flies, horn flies, and tabanids. Am. J. Vet. Res. 46, 123-126.

Elbers, A.R.W., Meiswinkel, R., Weezep, E., Oldruitenborgh-Oosterbaan, M.M.S. and Kooi, E.A. 2013. Schmallenberg virus in Culicoides spp. Biting Midges, the Netherlands, 2011. Emerg. Infect. Dis. J. 19, 106-109.

Foil, L.D. and Hogsette, J.A. 1994. Biology and control of tabanids, stable flies and horn flies. Rev. Sci. Tech. 13, 1125-1158.

Foil, L.D., Seger, C.L., French, D.D., Issel, C.J., McManus, J.M., Ohrberg, C.L. and Ramsey, R.T. 1988. Mechanical transmission of bovine leukemia virus by horse flies (Diptera: Tabanidae). J. Med. Entomol. 25, 374-376.

Hopkins, S.G. and DiGiacomo, R.F. 1997. Natural transmission of bovine leukemia virus in dairy and beef cattle. Vet. Clin. North Am. Food Anim. Pract. $13,107-128$.

Kobayashi, S., Hidano, A., Tsutsui, T., Yamamoto, T., Hayama, Y., Nishida, T., Muroga, N., Konishi, M., Kameyama, K. and Murakami, K. 2014. Analysis of risk factors associated with bovine leukemia virus seropositivity within dairy and beef breeding farms in Japan: A nationwide survey. Res. Vet. Sci. 96, 47-53.

Kohara, J., Konnai, S. and Onuma, M. 2006. Experimental transmission of Bovine leukemia virus in cattle via rectal palpation. Jpn. J. Vet. Res. $54,25-30$.

Kuramochi, K. 2000. Ovipositional behavior of the horn fly (Diptera: Muscidae) in the field. J. Med. Entomol. 37, 461-466.

Lagarias, D.M. and Radke, K. 1989. Transcriptional activation of bovine leukemia virus in blood cells from experimentally infected, asymptomatic sheep with latent infections. J. Virol. 63, 2099-2107.

Lassauzet, M.L.G., Thurmond, M.C., Johnson, W.O., Stevens, F. and Picanso, J.P. 1991. Factors associated with transmission of bovine leukemia virus by contact in cows on a California dairy. Am. J. Epidemiol. 133, 164-176.

Licursi, M., Inoshima, Y., Wu, D., Yokoyama, T., González, E.T. and Sentsui, H. 2003. Provirus variants of bovine leukemia virus in naturally infected cattle from Argentina and Japan. Vet. Microbiol. 96, 17-23.

Mullens, B.A. 2002. Horse Flies and Deer Flies (Tabanidae). In Medical and Veterinary Entomology. Eds., Mullen, G. and Durden, L. London, UK: Academic Press, pp: 263-277.

Ooshiro, M., Konnai, S., Katagiri, Y., Afuso, M., Arakaki, N., Tsuha, O., Murata, S. and Ohashi, K. 2013. Horizontal transmission of bovine leukemia virus from lymphocytotic cattle, and beneficial effects of insect vector control. Vet. Rec. 173, 527.

Oyarzún, M.P., Quiroz, A. and Birkett, M.A. 2008. Insecticide resistance in the horn fly: alternative control strategies. Med. Vet. Entomol. 22(3), 188202.

Panei, C.J., Takeshima, S.N., Omori, T., Nunoya, T., Davis, W.C., Ishizaki, H., Matoba, K. and Aida, Y. 2013a. Estimation of bovine leukemia virus (BLV) proviral load harbored by lymphocyte subpopulations in BLV-infected cattle at the subclinical stage of enzootic bovine leucosis using BLV-CoCoMo-qPCR. BMC Vet. Res. 9, 95; doi:10.1186/1746-6148-9-95

Panei, C.J., Serena M.S., Metz, G.E., Bravi, M.E., Gonzalez, E.T. and Echeverría, M.G. 2013 b. Analysis of the $\mathrm{pX}$ region of bovine leukemia virus in different clinical stages of the Enzoótic Bovine Leukemia in Argentinian Holstein. Virus Res. 171(1), 97-102.

Polat, M., Takeshima, S.N., Hosomichi, K., Kim, J., Miyasaka, T., Yamada, K., Arainga, M., Murakami, T., Matsumoto, Y., Barra Diaz, V., Panei, C.J., González, E.T., Kanemaki, M., Onuma, M., Giovambattista, G. and Aida, Y. 2016. A new genotype of bovine leukemia virus in South America identified by NGS-based whole genome sequencing and molecular evolutionary genetic analysis. Retrovirology 13, 4; doi:10.1186/s12977016-0239-Z

Pruett, J.H., Steelman, C.D., Miller, J.A., Pound, J.M. and George, J.E. 2003. Distribution of horn flies on individual cows as a percentage of the total horn fly population. Vet. Parasitol. 116(3), 251-258.

Sprecher, D.J., Pelzer, K. D. and Lessard, P. 1991 Possible effect of altered management practices on seroprevalence of bovine leukemia virus in heifers of a dairy herd with history of high prevalence of infection. J. Am. Vet. Med. Assoc. 199, 584. 